\title{
High-Pressure Gas TPC (HPgTPC) for DUNE Near Detector
}

\author{
Tanaz Angelina Mohayai \\ Physics Opportunities in the Near DUNE Detector Hall \\ Dec. 3, 2018
}




\section{Outline}

-Purpose

-Conceptual Design

-Expected Physics Performance

- $v$ Channels of Interest

-Summary \& Discussion 


\section{Outline}

\section{-Purpose}

-Conceptual Design

-Expected Physics Performance

- $v$ Channels of Interest

-Summary \& Discussion 


\section{Purpose}

Primary role is controlling the systematic uncertainties present in oscillation measurements - those dominated by cross-section, flux, \& $v$-energy

Other important roles:

\section{- As a component of the DUNE near detector:}

- Tag muons originating in ArgonCube

- Tag sign of charged particles exiting ArgonCube

- As a stand-alone magnetized spectrometer:

- In $v$-interactions in the gas, detect charged particles of very low energies

- Has superb:

$\star$ Tracking efficiency, PID

$\star$ Momentum \& angular resolution

- Magnetic field helps HPgTPC to:

$v$ beam

$\star$ Determine charge sign on an event-by-event basis \& discriminate between $v / \bar{v}$

$\star$ A background-free sample of $v_{\mathrm{e}} \mathrm{CC}$ events via sign tagging in b-field

- As a tracker surrounded by the ECAL calorimeter:

- Detect neutrons \& tag exiting particles 


\section{Purpose}

Primary role is controlling the systematic uncertainties present in oscillation measurements - those dominated by cross-section, flux, \& $v$-energy

Other important roles:

\section{- As a component of the DUNE near detector:}

- Tag muons originating in ArgonCube

- Tag sign of charged particles exiting ArgonCube

- As a stand-alone magnetized spectrometer:

c In $v$-interactions in the gas, detect charged particles of very low energies

- Has superb:

$\star$ Tracking efficiency, PID

$\star$ Momentum \& angular resolution

- Magnetic field helps HPgTPC to:

$v$ beam

$\star$ Determine charge sign on an event-by-event basis \& discriminate between $v / \bar{v}$

$\star$ A background-free sample of $v_{\mathrm{e}} \mathrm{CC}$ events via sign tagging in b-field

- As a tracker surrounded by the ECAL calorimeter:

- Detect neutrons \& tag exiting particles 


\section{Outline}

-Purpose

-Conceptual Design

-Expected Physics Performance

- $v$ Channels of Interest

-Summary \& Discussion 


\section{HPgTPC Conceptual Design}

- Copy of the ALICE TPC:

- Will reuse 72 ALICE Inner and Outer Readout Chambers (IROC and OROC):

$\star$ Available because of planned ALICE upgrade, a significant cost reduction for DUNE

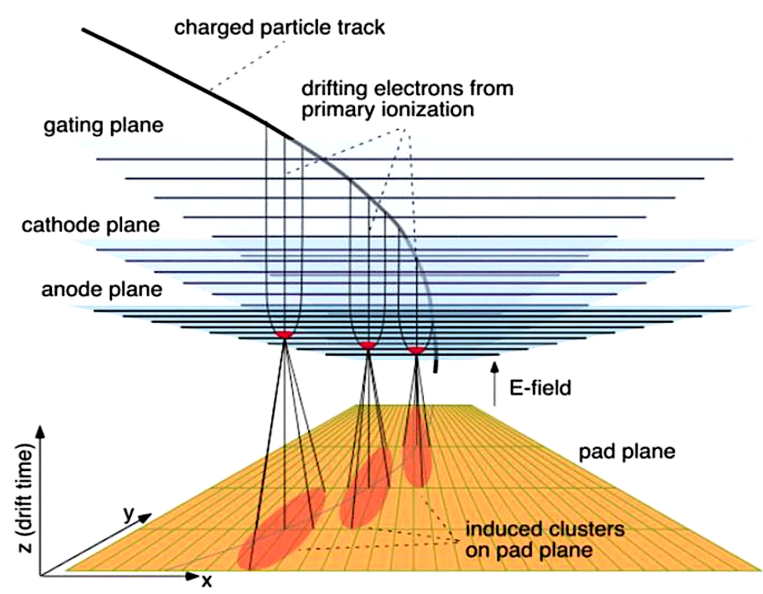

$\star$ Operated @ 1 atm pressure in ALICE. Will operate @ 10 atm pressure in DUNE HPgTPC

- Primary gas mixture in DUNE HPgTPC will be Ar$\mathrm{CH}_{4}$ (P10 with $97 \%$ of interactions on $\mathrm{Ar}$ ):

- Possible to study other nuclei such as $\mathrm{H}_{2}$ (safety concern but not impossible!), $\mathrm{D}_{2}, \mathrm{Ne}, \mathrm{CF}_{4}, \mathrm{Xe}$

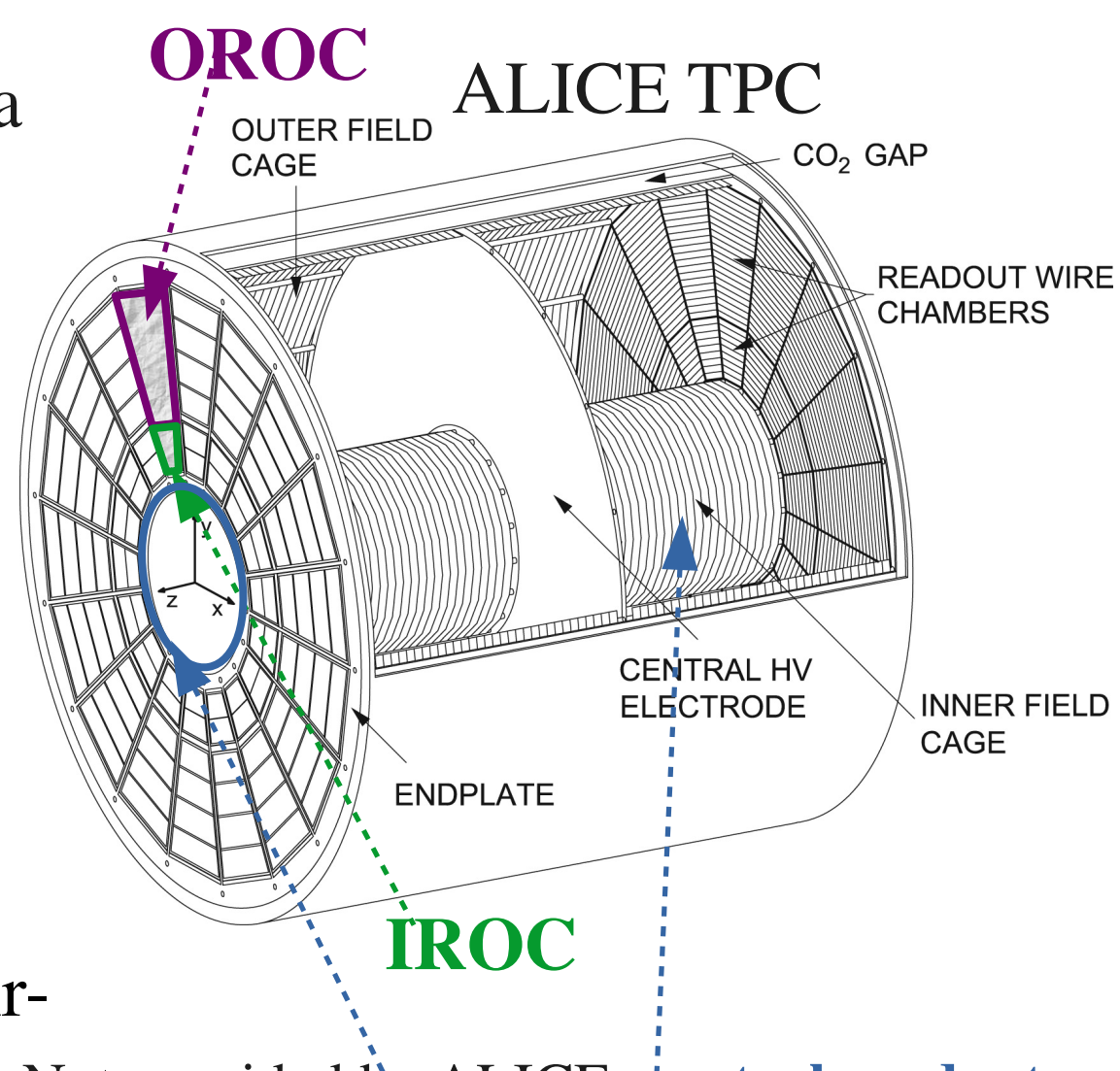

Not provided by ALICE: central readout chambers (do not exist in ALICE), field cages, front-end electronics
2018-12-03
T. A. Mohayai 


\section{HPgTPC Test Stand @ FNAL}

- Gaseous-Argon Operation of the ALICE TPC, GOAT

- Test ALICE readout chambers at $10 \mathrm{~atm}$ and in various gas mixture (currently 90-10 Ar$\mathrm{CO}_{2}$ )

- Develop full front-end electronics chain

- Various components in GOAT:

- Signal readout with ALICE IROC

- Field cage

- Front-end with preamps and CAEN digitizers

- Upgrades to components underway; stay tuned!

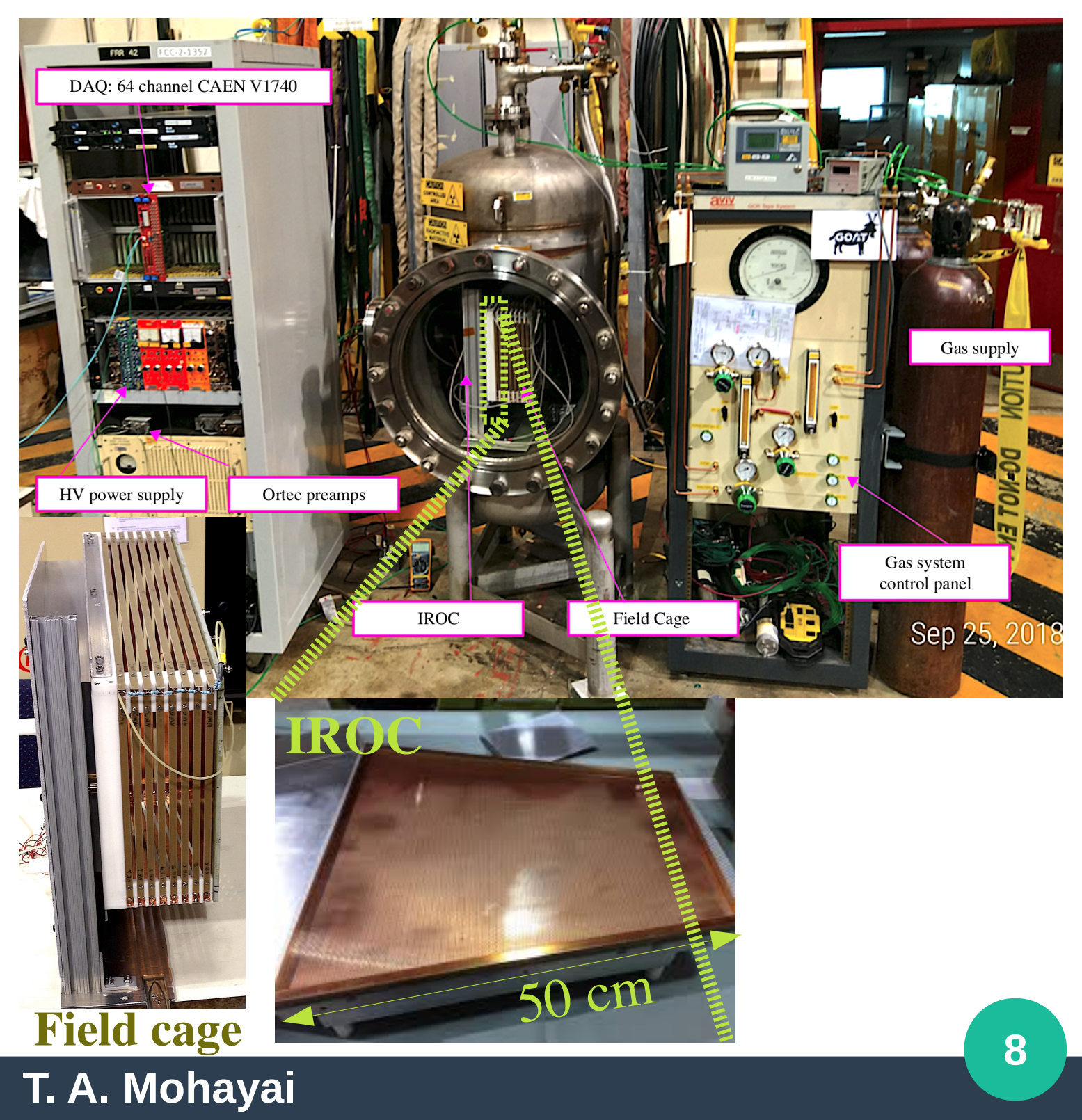




\section{Conceptual Design - HPgTPC ECAL}

- An electromagnetic calorimeter, inspired by the CALICE calorimeter design:

- Made of plastic scintillators (readout by silicon photomultipliers) sandwiched between lead absorber sheets

$20-30 \mathrm{~mm}$

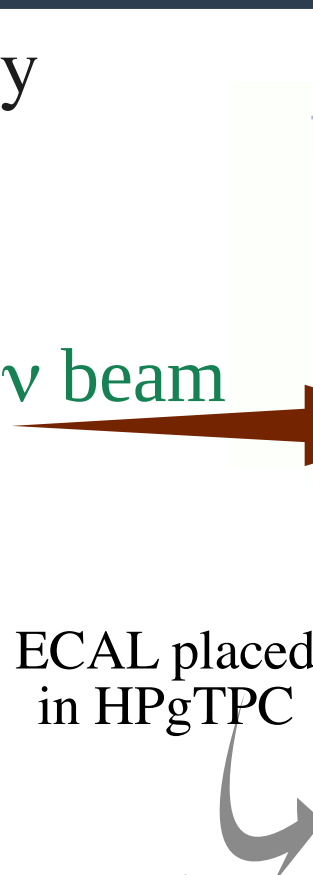

F. Simon, E. Brianne

$\mathrm{Pb}$ absorber $\square$ plastic scintillator SiPM $\square$ signal/power routing

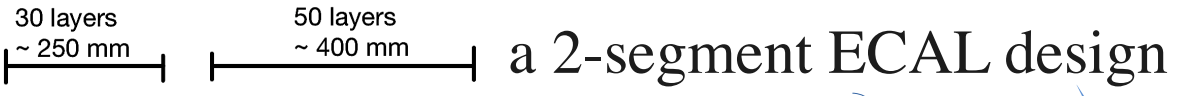

- Factors affecting its design:

- Limited space inside the pressure vessel $\rightarrow$ possibly needs a 2-segment design

- Good directional resolution $\rightarrow$ high granular with a high sampling frequency
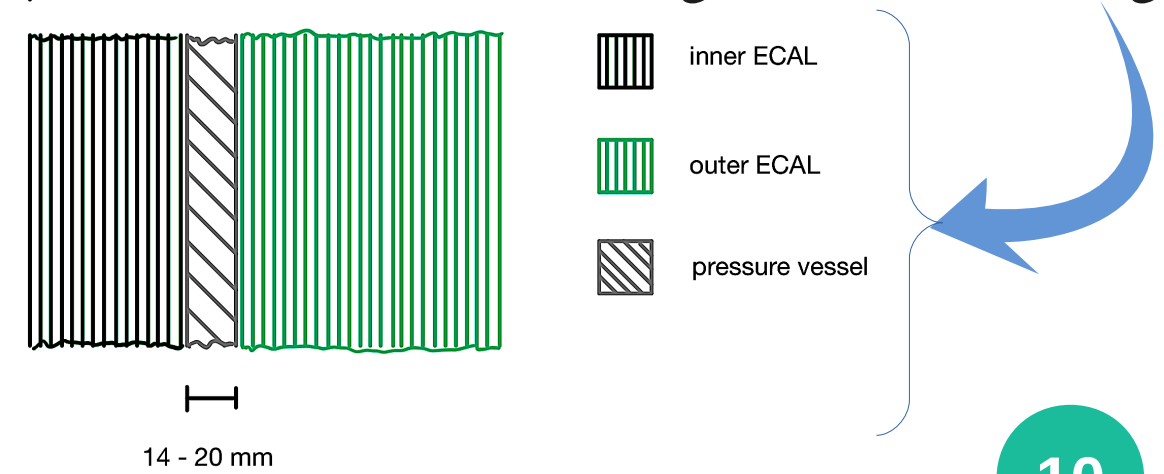


\section{Outline}

-Purpose

-Conceptual Design

-Expected Physics Performance

- $v$ Channels of Interest

- Summary \& Discussion 


\section{HPgTPC Physics Role}

- Crucial to understand $v$ - $\mathrm{N}$ interactions to accurately reconstruct $\boldsymbol{v}$-energy $\&$ cross-section

- Nucleus is a complicated environment:

- Experimental data limited in nuclear targets \& no data in lpw $\boldsymbol{v}$-energy

- HPgTPC helps:

- Lower density $\left(\rho_{\mathrm{LAr}} / \rho_{\mathrm{GAr}} \approx 85\right.$ for 10 atm GAr)

$\rightarrow$ lower detection threshold $\rightarrow$ higher sensitivity to charged particles at lower energies
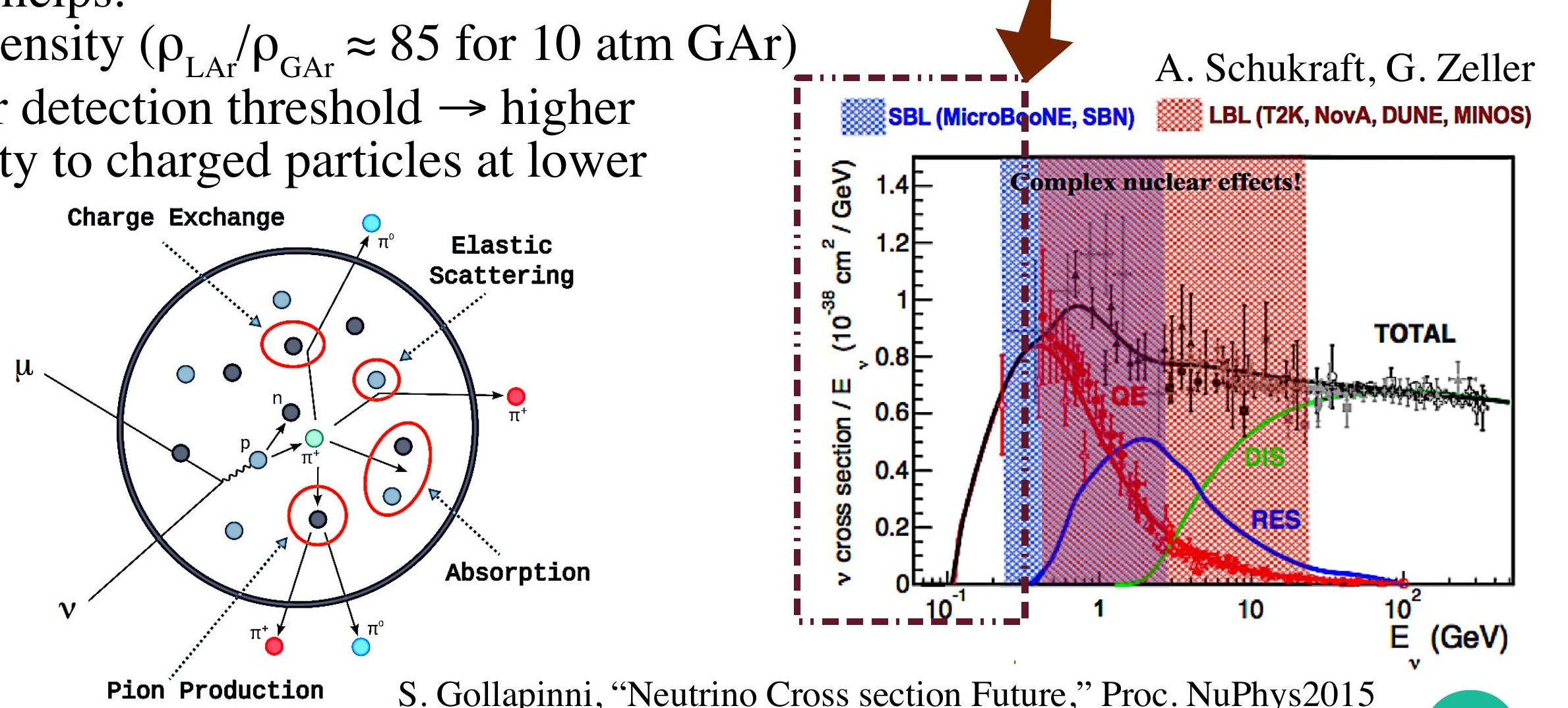

Pion Production

S. Gollapinni, "Neutrino Cross section Future," Proc. NuPhys2015 


\section{HPgTPC Physics Role}

- In addition, need to understand discrepancies between event generators at lower energies

- Lower detection threshold (than in LAr) in HPgTPC is critical for this
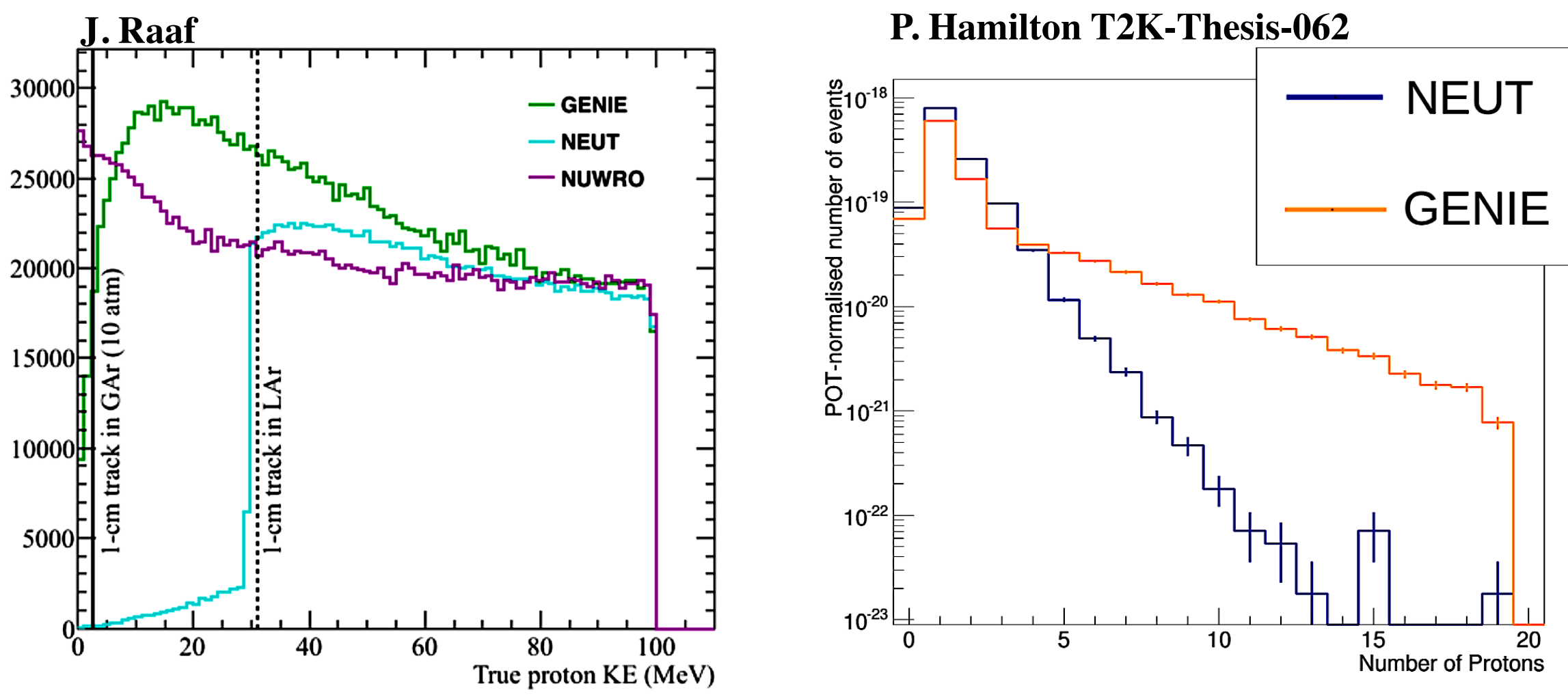


\section{Expected Physics Performance}

- Event displays of proton and electron tracks (some are final state particles from $v$-N interactions) inside the HPgTPC

$30 \mathrm{MeV}$ electron traveling a distance of $\sim 6 \mathrm{~m}$

a display of proton tracks

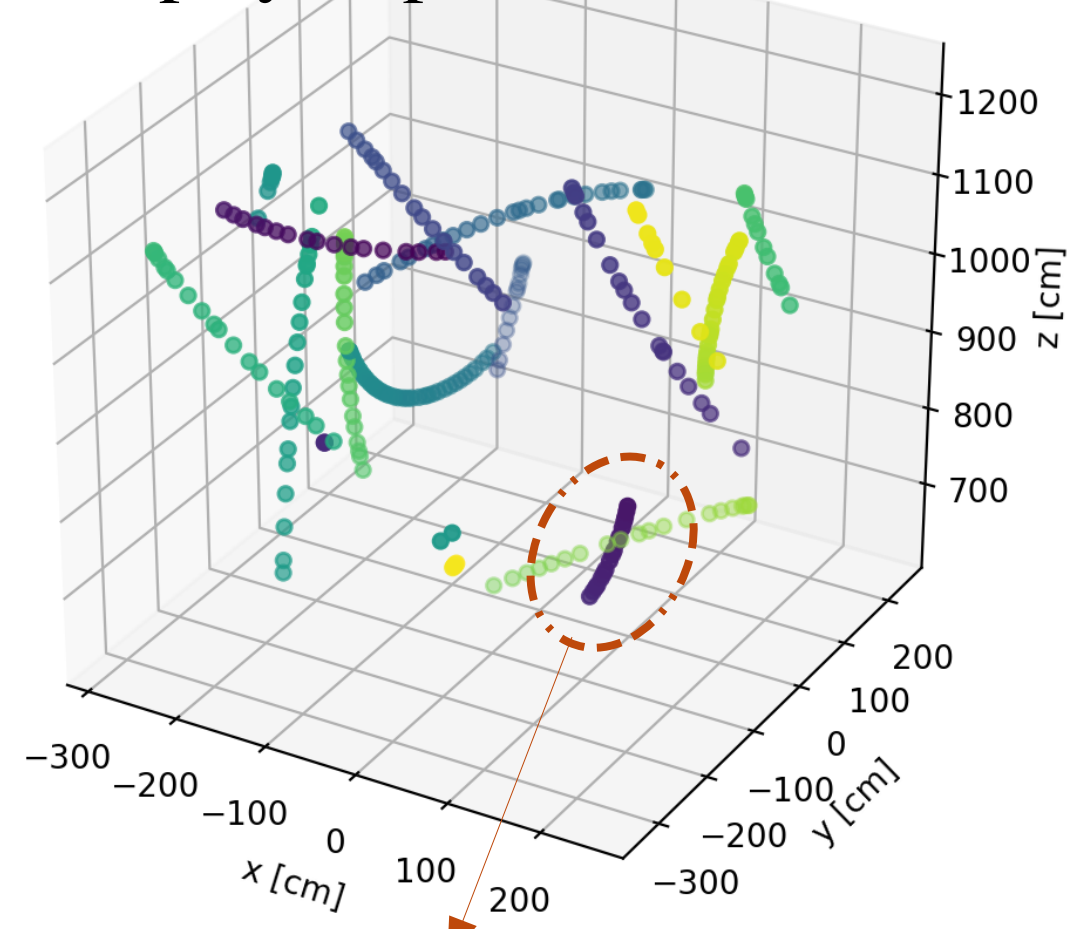

$40 \mathrm{MeV}$ proton with range of $\sim 1 \mathrm{~m}$

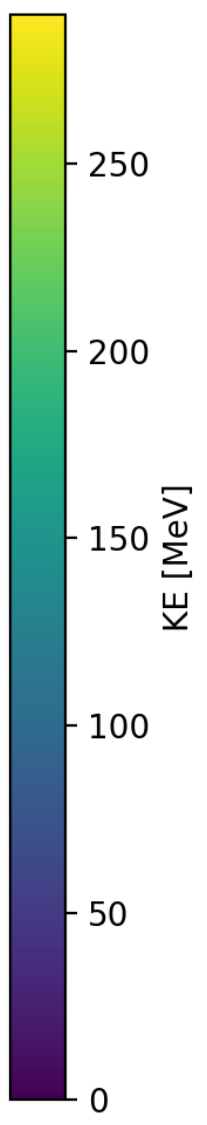

T. A. Mohayai

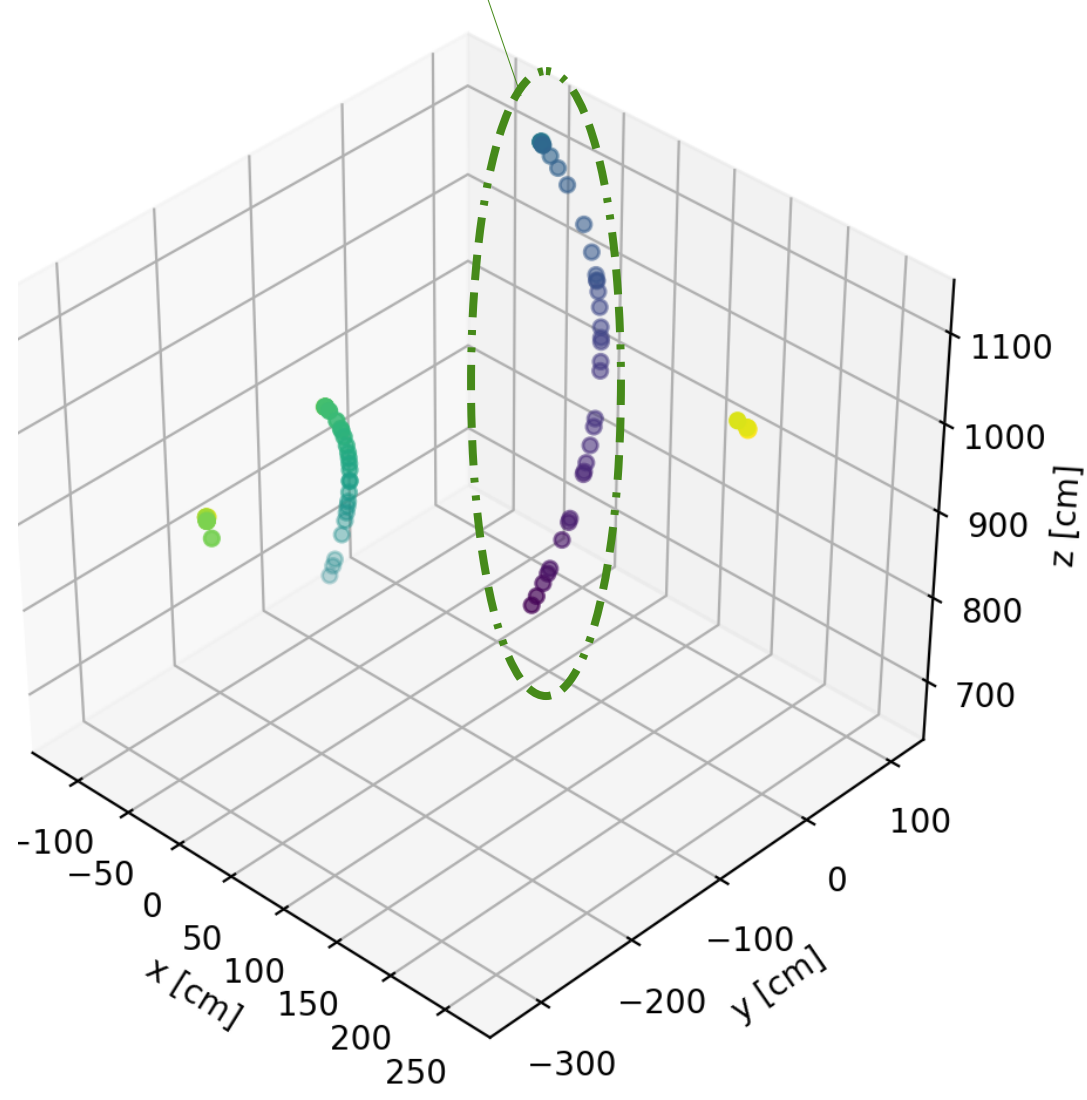

a display of electron tracks
80

70

60

$50 \frac{2}{\sum_{0}^{2}}$

30

20 


\section{Expected Physics Performance}

- A $4 \pi$ coverage \& excellent tracking efficiency (based on ALICE performance)

- High multiplicity in HPgTPC will not be an issue - hint: take a look at the ALICE events
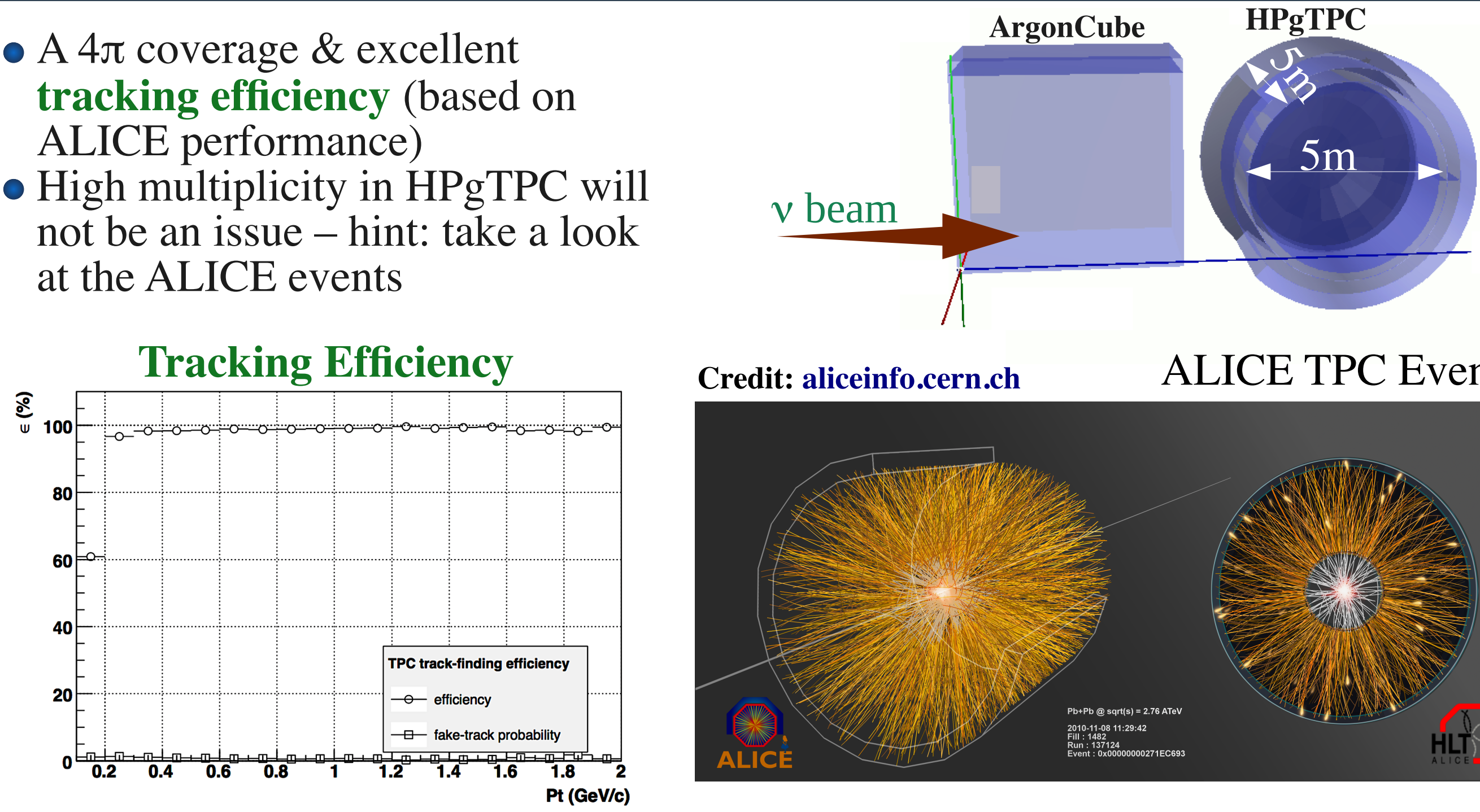

Credit: aliceinfo.cern.ch

ALICE TPC Events

C. W. Fabjan et al. (ALICE), J. Phys. G32, 1295 (2006)
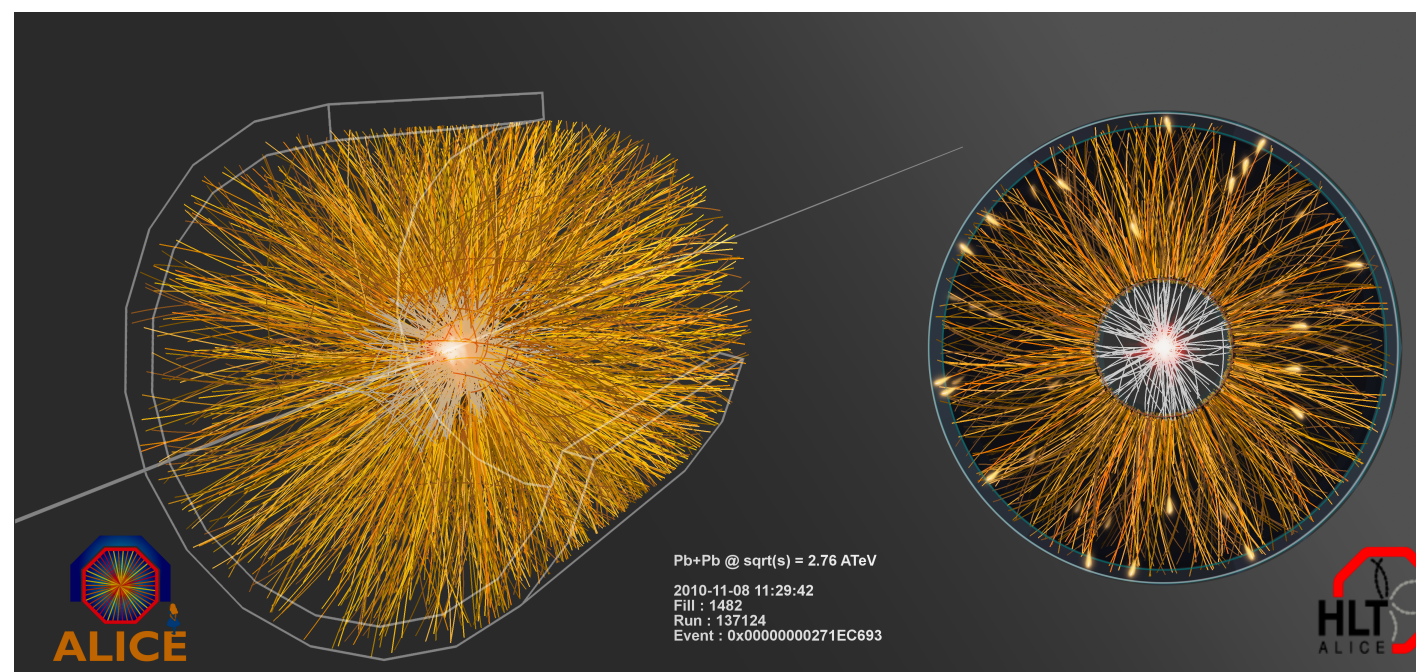


\section{Expected Physics Performance}

- Excellent PID based on ALICE \& PEP-4 results - HPgTPC will operate at even higher pressure (10 atm pressure) than PEP-4 (8.5 atm pressure) $\rightarrow$ even better PID

- Clear distinction between particles, in particular at lower momenta

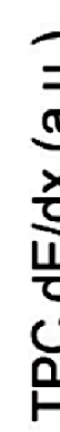
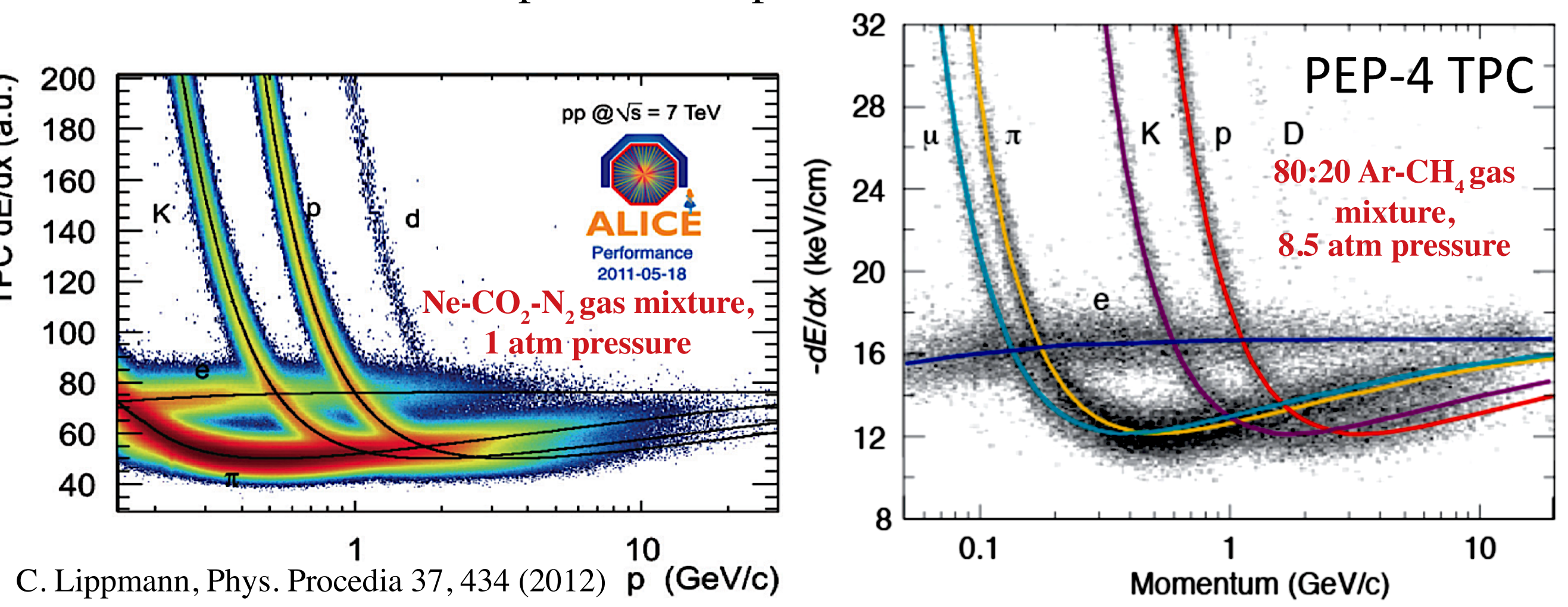


\section{Expected Physics Performance}

- Performance parameters based on ALICE \& PEP-4:

- Less multiple scattering in gas (a limiting factor in momentum resolution) $\rightarrow$ great momentum (black squares in momentum resolution plot) \& angular resolutions

\begin{tabular}{|c|c|c|}
\hline Parameter & Value & units \\
\hline$\sigma_{x}$ & 250 & $\mu \mathrm{m}$ \\
\hline$\sigma_{y}$ & 250 & $\mu \mathrm{m}$ \\
\hline$\sigma_{z}$ & 1500 & $\mu \mathrm{m}$ \\
\hline$\sigma_{r \phi}$ & $<1000$ & $\mu \mathrm{m}$ \\
\hline Two-track separation & 1 & $\mathrm{~cm}$ \\
\hline Angular resolution & $\underline{2}-\overline{4}$ & mrad \\
\hline$\sigma(\mathrm{dE} / \mathrm{dx})$ & 5 & $\%$ \\
\hline $\bar{\sigma}_{p_{T}}^{-} / \bar{p}_{T}$ & 0.7 & $--\overline{\%} \cdot(\overline{10}-1 \overline{\mathrm{GeV}} / \mathrm{c})$ \\
\hline$\sigma_{p_{T}} / p_{T} \ldots-\ldots--$ & $\underline{1-2}$ & $\%(1-0.1 \mathrm{GeV} / \mathrm{c})$ \\
\hline Energy scale uncertainty & $\lesssim 1$ & $\%$ (dominated by $\left.\delta_{p} / p\right)$ \\
\hline Charge particle detection thresh. & 5 & $\mathrm{MeV}($ K.E. $)$ \\
\hline ECAL resolution & $5-7 / \sqrt{E / G e V}$ & $\%$ \\
\hline ECAL pointing resolution & $\simeq 6$ at $500 \mathrm{MeV}$ & degree \\
\hline
\end{tabular}

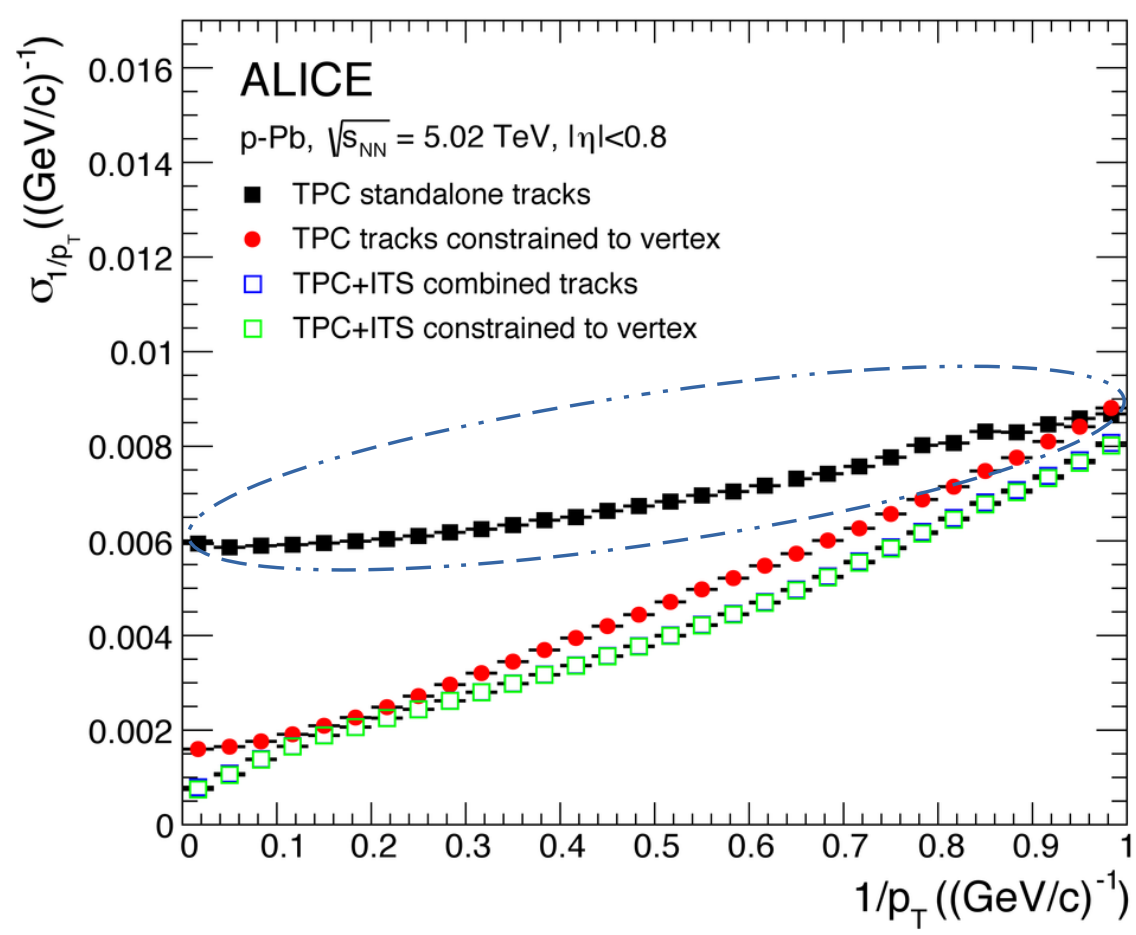

B. B. Abelev et al. (ALICE), Int. J. Mod. Phys. A29, 1430044 (2014), 1402.4476 


\section{Expected Physics Performance}

- Parameters used in determining ECAL performance:

- Energy \& angular resolution - obtained using:

$\star$ GEANT-4 based simulation, simplified detector model, simplified reconstruction \& single photon energies

$\star$ A 2-segmented ECAL design
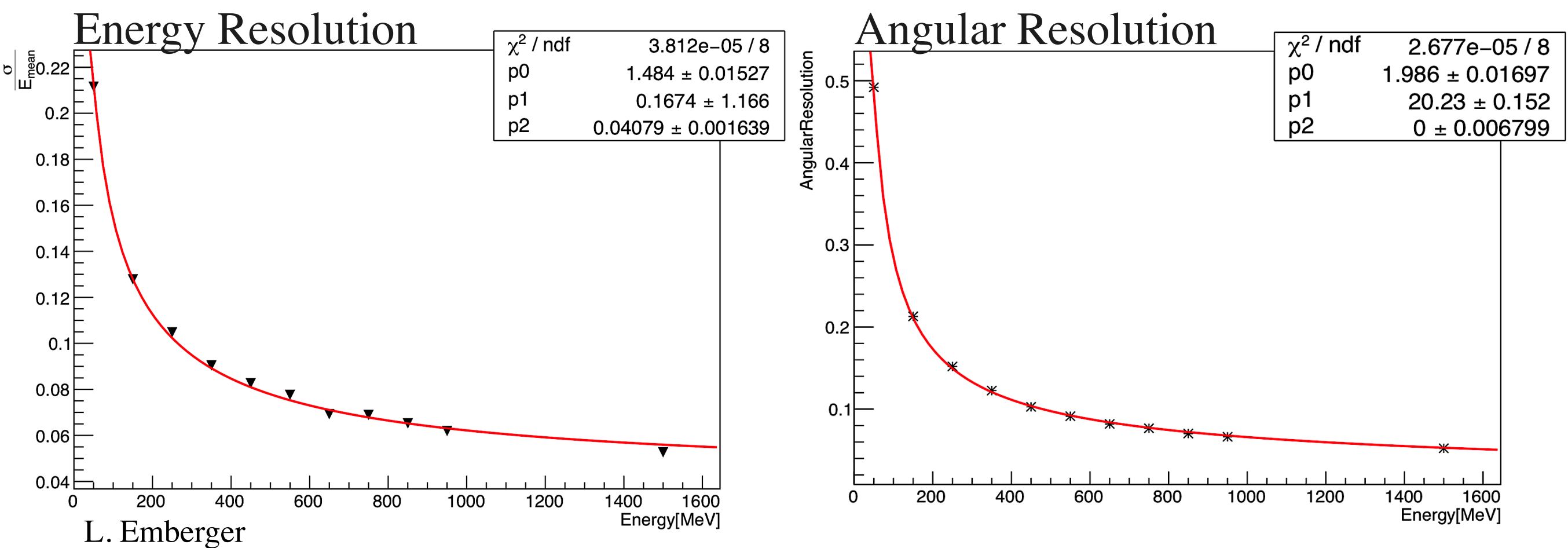


\section{Outline}

-Purpose

-Conceptual Design

- Expected Physics Performance

- $v$ Channels of Interest

-Summary \& Discussion 


\section{Key $v$ channels}

- Some standard $v$ channels $\&$ their stats

- Event display from $v_{\mu} \mathrm{CC}$ interaction:

- GENIE event generator to generate the $v$-interactions + GEANT4-based simulation to reconstruct the energy $(v$-energy of $\sim 1 \mathrm{GeV}$ )

\begin{tabular}{|c|c|}
\hline Event class & Number of events per ton-year \\
\hline$v_{\mu}$ CC Total & $1.64 \times 10^{6}$ \\
\hline$v_{\mu}$ NC Total & $5.17 \times 10^{5}$ \\
\hline$v_{\mu}$ CC Coherent & $8.35 \times 10^{3}$ \\
\hline$v_{\mu}$ NC Coherent & $4.8 \times 10^{3}$ \\
\hline$v_{\mu}$ - electron elastic & 135 \\
\hline$v_{\mu} \mathrm{CC} \pi^{0}$ inclusive & $4.47 \times 10^{5}$ \\
\hline$\underline{v}_{\mu} \mathrm{NC} \pi^{0}$ inclusive & $1.96 \times 10^{5}$ \\
\hline$v_{\mu}$ Low $\mathrm{v}(250 \mathrm{MeV})$ & $2.16 \times 10^{5}$ \\
\hline$\nu_{\mu}$ Low $\mathrm{v}(100 \mathrm{MeV})$ & $7.93 \times 10^{4}$ \\
\hline $\bar{v}_{\mu} \mathrm{CC}$ Coherent $(\bar{v}$ mode $)$ & $6.90 \times 10^{3}$ \\
\hline$v_{e}$ CC Total & $1.89 \times 10^{4}$ \\
\hline$v_{e}$ NC Total & $5.98 \times 10^{3}$ \\
\hline$v_{e}$ CC Coherent & 93 \\
\hline$v_{e}$ NC Coherent & 52 \\
\hline
\end{tabular}

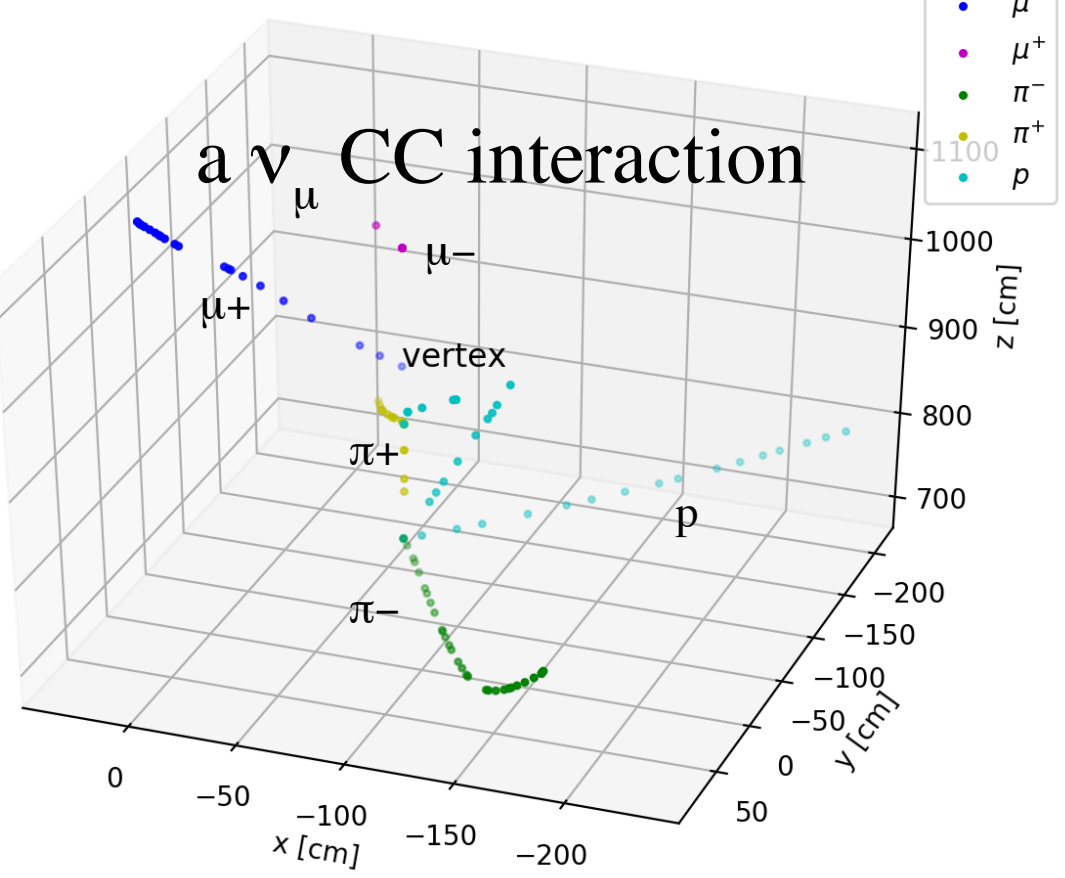




\section{Key v channels}

- As a magnetized tracker, HPgTPC can:

- Obtain a background-free sample of $v_{e}$ $\mathrm{CC}$ events via wrong-sign tagging in b-fied

- In LArTPC:

- $v_{\mu} \mathrm{NC} \pi^{0} \mathrm{~s}$ are misidentified as $\nu_{\mathrm{e}} \mathrm{CCs}$

- In HPgTPC, not an issue:

- No $\pi^{0} \mathrm{~s}$ conversion in gas

- Most NC $\pi^{0}$ events easily tagged by oppositely-bending $\mathrm{e}^{+}$and $\mathrm{e}^{-}$tracks

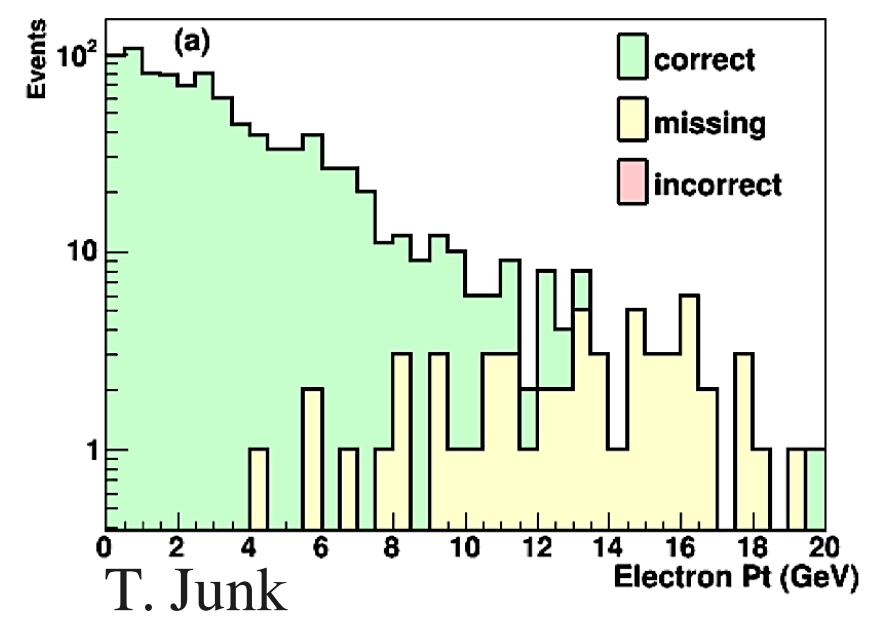

\begin{tabular}{|c|c|}
\hline Event class & Number of events per ton-year \\
\hline$v_{\mu}$ CC Total & $1.64 \times 10^{6}$ \\
\hline$v_{\mu} \mathrm{NC}$ Total & $5.17 \times 10^{5}$ \\
\hline$v_{\mu} \mathrm{CC}$ Coherent & $8.35 \times 10^{3}$ \\
\hline$v_{\mu} \mathrm{NC}$ Coherent & $4.8 \times 10^{3}$ \\
\hline$v_{\mu}$ - electron elastic & 135 \\
\hline$v_{\mu} \mathrm{CC} \pi^{0}$ inclusive & $4.47 \times 10^{5}$ \\
\hline$v_{\mu} \mathrm{NC} \pi^{0}$ inclusive & $1.96 \times 10^{5}$ \\
\hline$v_{\mu}$ Low v $(250 \mathrm{MeV})$ & $2.16 \times 10^{5}$ \\
\hline$v_{\mu}$ Low v $(100 \mathrm{MeV})$ & $7.93 \times 10^{4}$ \\
\hline $\bar{v}_{\mu}$ CC Coherent ( $\bar{v}$ mode $)$ & $6.90 \times 10^{3}$ \\
\hline I $v_{e}$ CC Total & $1.89 \times 10^{4}$ \\
\hline$v_{e}$ NC Total & $5.98 \times 10^{3}$ \\
\hline$v_{e}$ CC Coherent & 93 \\
\hline$v_{e}$ NC Coherent & 52 \\
\hline
\end{tabular}

T. Junk 


\section{Key v channels}

- $\mathrm{CC} \pi^{+/-}$coherent scattering is a channel of interest:

- Same cross-section for $v$ and $\bar{v} \rightarrow$ can check for any biases in the two running modes

- Almost no energy transfer to nucleus $\rightarrow$ estimate true $v$-energy for both $v \& \bar{v}$

\begin{tabular}{|l|c|}
\hline & $\begin{array}{c}\text { Coherent-like } \\
\text { Events (Fraction passing cuts) }\end{array}$ \\
\hline \hline All non-coherent CC events (no cuts) & $745720(1.0)$ \\
LArTPC $\left(E_{\text {thresh }}=40 \mathrm{MeV}\right)$ & $407(0.0005)$ \\
$\operatorname{HPgTPC}\left(E_{\text {thresh }}=5 \mathrm{MeV}\right)$ & $8\left(1 \times 10^{-5}\right)$ \\
$\operatorname{HPgTPC}\left(E_{\text {thresh }}=2.5 \mathrm{MeV}\right)$ & $1\left(1 \times 10^{-6}\right)$ \\
\hline
\end{tabular}

- A cleaner sample can be selected with HPgTPC (thanks to its the low threshold) than LArTPC

Tingjun Yang et al. (ArgoNeuT collaboration) "First Measurement of Neutrino and Antineutrino Coherent Charged Pion Production on Argon," Phys. Rev. Lett. 113,261801 (2014)

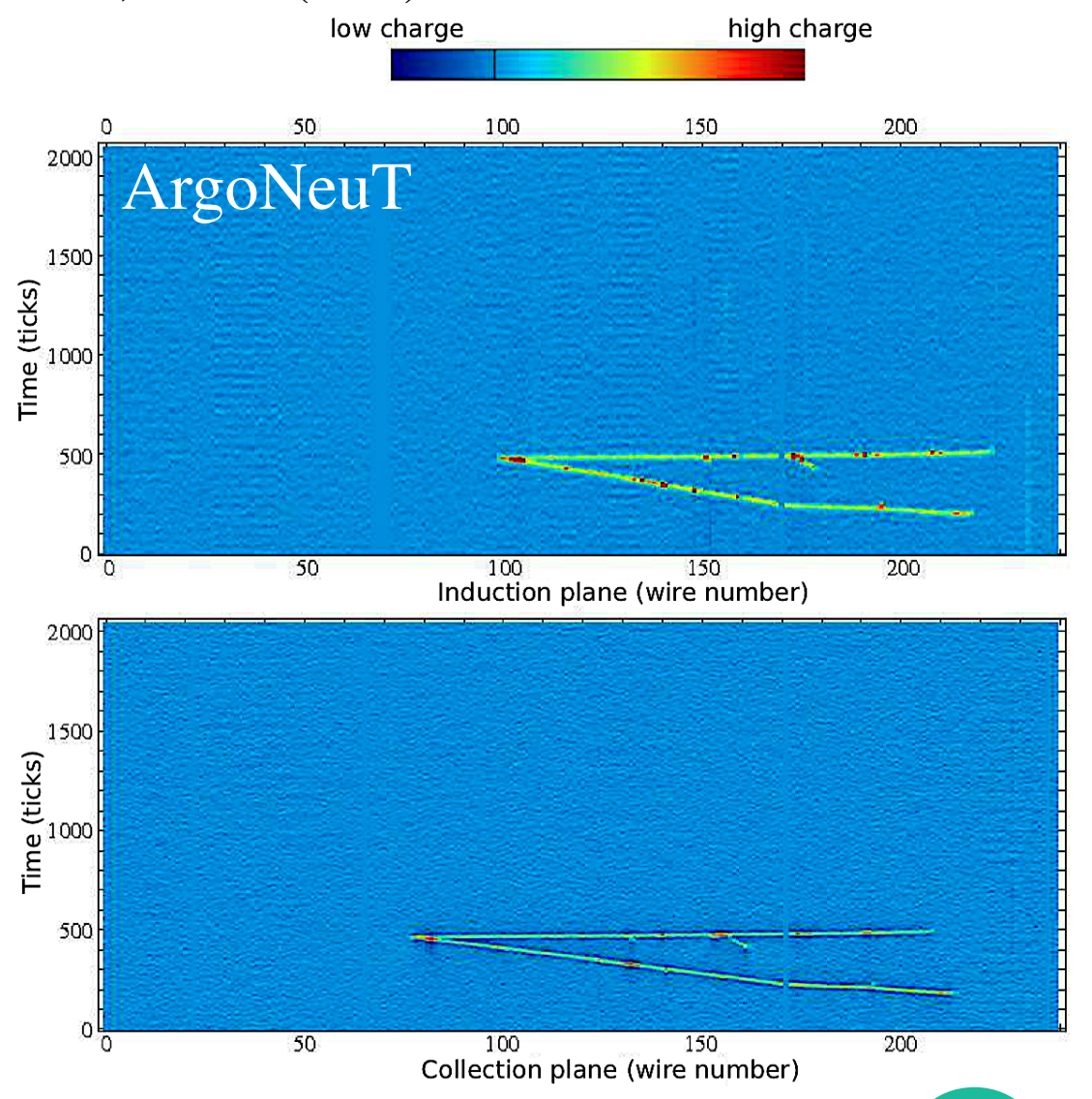




\section{Outline}

-Purpose

-Conceptual Design

-Expected Physics Performance

- v Channels of Interest

-Summary \& Discussion 


\section{Summary \& Discussion}

- The aim of the full near detector suite is to reduce the systematic uncertainties in the oscillation measurement to a few \% level:

- Main sources of uncertainty are measurements of cross-section, flux, and v-energy

- The HPgTPC is a crucial component of the near detector suite:

- Augment upstream detector by tracking and sign-tagging particles exiting LArTPC

- Collect independent sample of neutrino interactions on argon

- Extend neutrino cross section measurements to lower energies in region where data are sparse

- Background-free samples of CC coherent and intrinsic beam $v_{\mathrm{e}}$

- Test \& tune generator models at lower energies

- Capable of operating with other nuclear target materials $\left(\mathrm{H}_{2}, \mathrm{D}_{2}, \ldots\right)$

- The HPgTPC may also provide opportunities to search for exotic physics

- Milli-charged particles? Dark matter?... let's discuss! 
On behalf of the HPgTPC team: L. Bellantoni, E. Brianne, A. Bross, K. Duffy, G. Fernandez Moroni, T. Junk, J. Martin-Albo, T. Mohayai, J. Raaf

\section{Thank You!}

More collaborators are welcome! Contact us if interested! 


\section{Additional Slides}




\section{Near Detector Physics Motivation}

- Reducing the systematic uncertainties in the oscillation measurement to a few \% level: - Main sources of uncertainties are measurements of cross-section, flux, \& v-energy
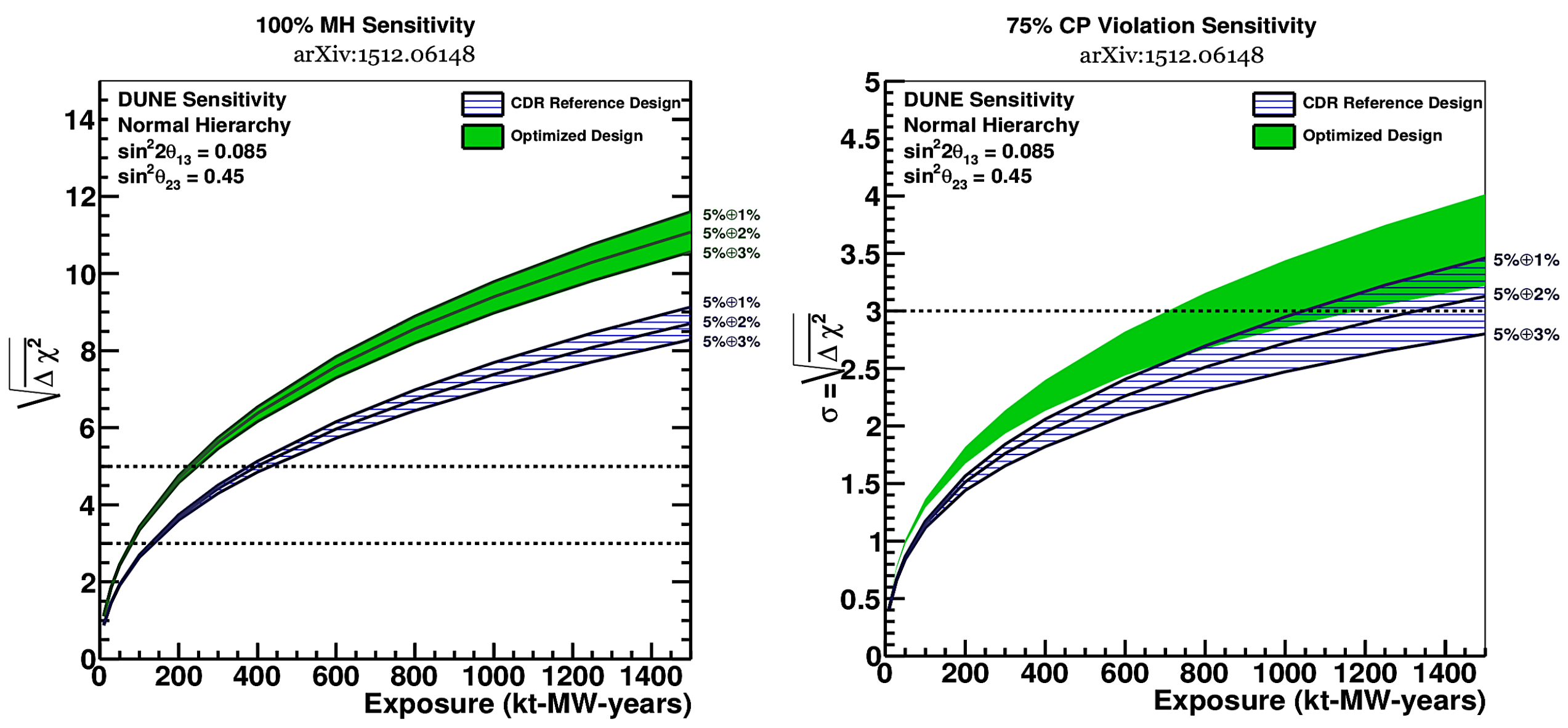


\section{Near Detector Physics Motivation}

- Reducing the systematic uncertainties in the oscillation measurement to a few $\%$ level:

- Main sources of uncertainties are measurements of cross-section, flux, \& v-energy - Observable is disappearance/appearance events vs. the $\boldsymbol{v}$-energy

a simplified oscillation measurement, from an experimental point of view:

$$
\begin{gathered}
P_{\nu_{\alpha} \rightarrow \nu_{\alpha^{\prime}}} \approx \frac{N_{\nu_{\alpha^{\prime}}}^{F D}\left(E_{\nu}\right)}{N_{\nu_{\alpha}}^{N D}\left(E_{\nu}\right)} \times \frac{\epsilon^{N D}\left(E_{\nu}\right)}{\epsilon^{F D}\left(E_{\nu}\right)} \\
\frac{N_{\nu_{\alpha^{\prime}}}^{F D}\left(E_{\nu}\right)}{N_{\nu_{\alpha}}^{N D}\left(E_{\nu}\right)}=\frac{\int \Phi_{\nu_{\alpha^{\prime}}}\left(E_{\nu}\right) \sigma_{\nu_{\alpha^{\prime}}}\left(E_{\nu}\right) d E_{\nu}}{\int \Phi_{\nu_{\alpha}}\left(E_{\nu}\right) \sigma_{\nu_{\alpha}}\left(E_{\nu}\right) d E_{\nu}}
\end{gathered}
$$




\section{Near Detector Physics Motivation}

- Reducing the systematic uncertainties in the oscillation measurement to a few $\%$ level:

- Main sources of uncertainties are measurements of cross-section, flux, \& v-energy - Observable is disappearance/appearance events vs. the $\boldsymbol{v}$-energy

a simplified oscillation measurement, from an experimental point of view:

$$
\begin{aligned}
& P_{\nu_{\alpha} \rightarrow \nu_{\alpha^{\prime}}} \approx \frac{N_{\nu_{\alpha^{\prime}}}^{F D}\left(E_{\nu}\right)}{N_{\nu_{\alpha}}^{N D}\left(E_{\nu}\right)} \times \frac{\epsilon^{N D}\left(E_{\nu}\right)}{\epsilon^{F D}\left(E_{\nu}\right)}
\end{aligned}
$$

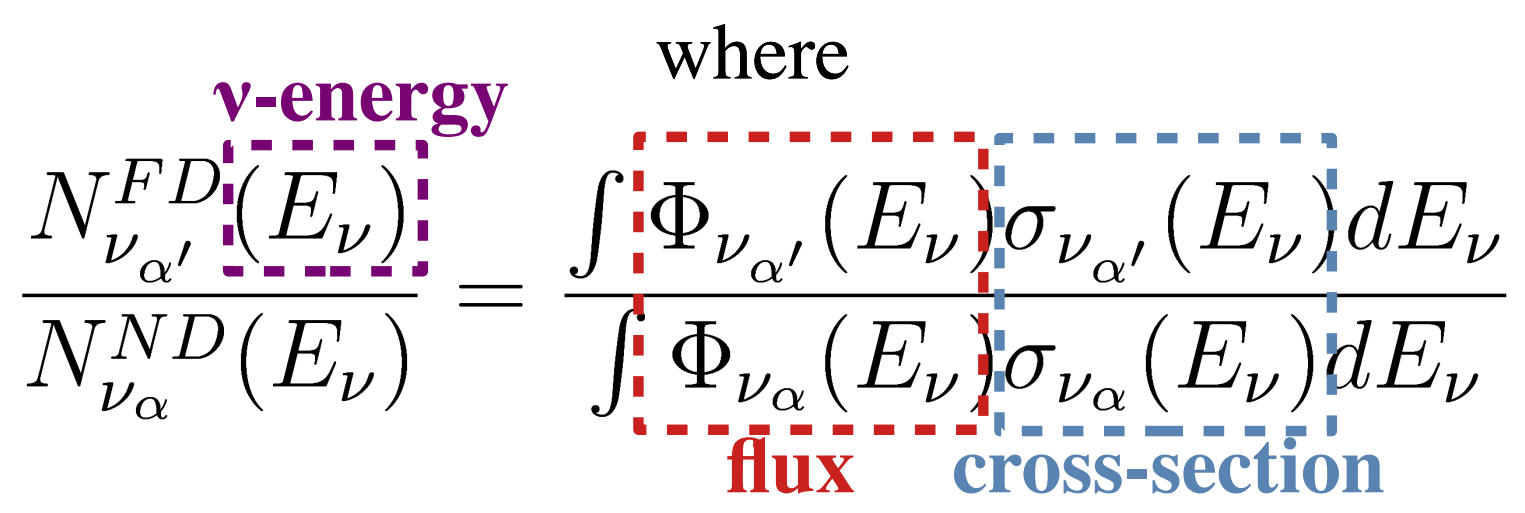




\section{Near Detector Physics Motivation}

- Reducing the systematic uncertainties in the oscillation measurement to a few \% level: - Main sources of uncertainties are measurements of cross-section, flux, \& v-energy - Observable is disappearance/appearance events vs. the $\boldsymbol{v}$-energy
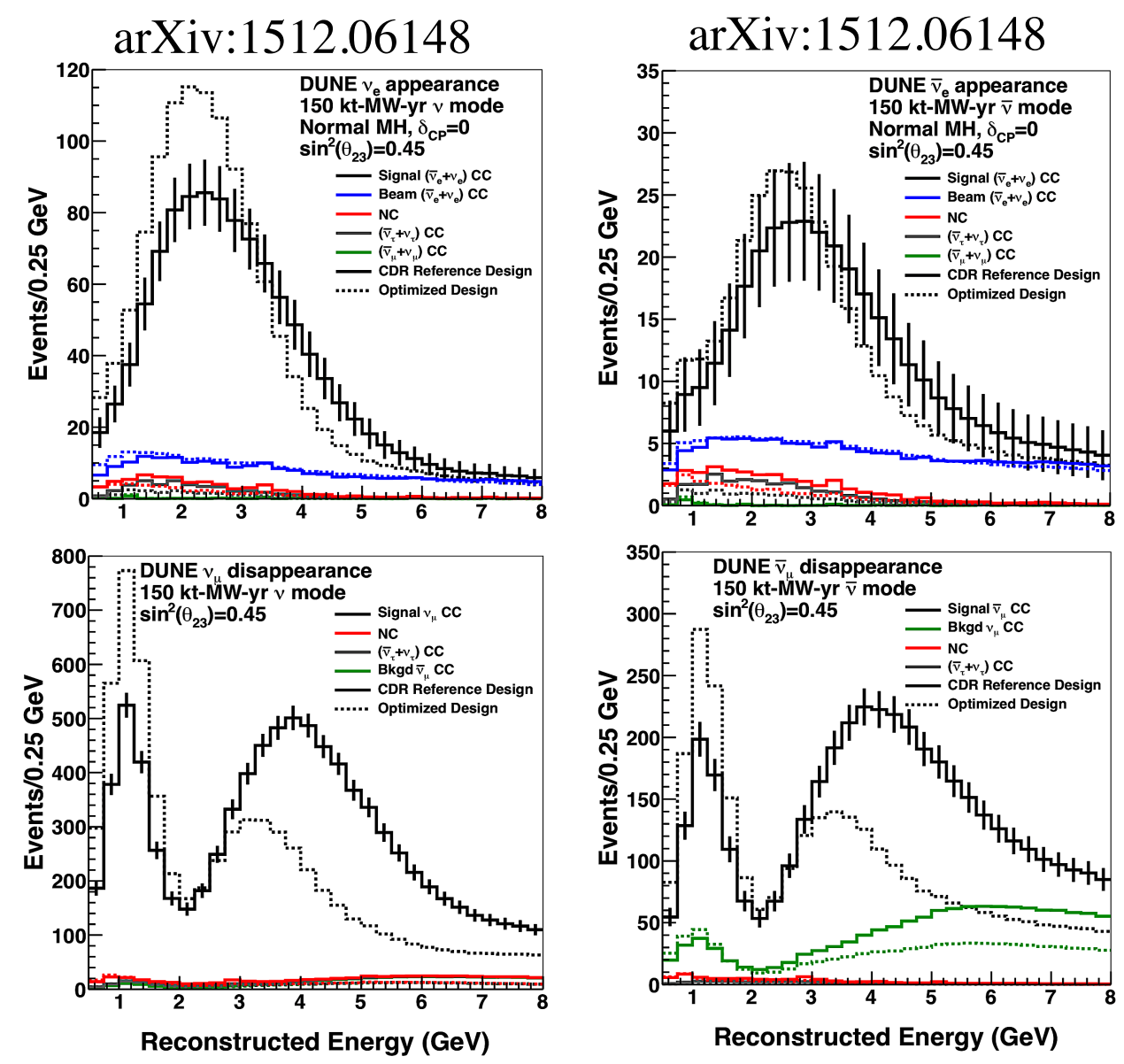